\title{
Oral versus intubated feeding and the effect on glycaemic and insulinaemic responses, gastric emptying and satiety
}

Article

Accepted Version

Creative Commons: Attribution-Noncommercial-No Derivative Works 4.0

Morey, S., Shafat, A. and Clegg, M. E. (2016) Oral versus intubated feeding and the effect on glycaemic and insulinaemic responses, gastric emptying and satiety. Appetite, 96. pp. 598-603. ISSN 0195-6663 doi:

https://doi.org/10.1016/j.appet.2015.11.002 Available at https://centaur.reading.ac.uk/80390/

It is advisable to refer to the publisher's version if you intend to cite from the work. See Guidance on citing.

To link to this article DOI: http://dx.doi.org/10.1016/j.appet.2015.11.002

Publisher: Elsevier

All outputs in CentAUR are protected by Intellectual Property Rights law, including copyright law. Copyright and IPR is retained by the creators or other copyright holders. Terms and conditions for use of this material are defined in the End User Agreement.

www.reading.ac.uk/centaur 
Central Archive at the University of Reading

Reading's research outputs online 
1 Oral versus intubated feeding and the effect on glycaemic and insulinaemic responses, gastric

2 emptying and satiety.

3

4 Morey $\mathrm{S}^{\mathrm{a}}$, Shafat $\mathrm{A}^{\mathrm{b}}$ and Clegg M. $\mathrm{E}^{\mathrm{a}}$

5

6 a Functional Food Centre, Department of Sport and Health Sciences, Faculty of Health and Life

7 Sciences, Oxford Brookes University, Gipsy Lane, Oxford OX3 0BP, UK

$8 \quad{ }^{b}$ Physiology, School of Medicine, National University of Ireland, Galway, Ireland

9

10 Corresponding author: Dr Miriam E Clegg, Functional Food Centre,

11 Department of Sport and Health Sciences, Faculty of Health and Life Sciences, Oxford Brookes

12 University, Gipsy Lane, Oxford OX3 0BP, UK

13 Email: mclegg@ brookes.ac.uk; Ph: +44 1865484365

14

15

16

17

18

19

20

21

22

23 


\section{Abstract}

26 Cephalic phase responses (CPR) are important in early initiation of digestion and maximal

27 absorption of nutrients prior to ingestion. Bypassing CPR has been shown to have consequences

28 on metabolic responses that may influence satiety. The aim of this study was to investigate if

29 using gastric intubation to bypass oro-pharyngeal and oesophageal exposure would reduce CPR

30 including insulin and blood glucose and whether these impact on gastric emptying and satiety.

31 Ten male subjects were tested on 2 occasions, 3-7 days apart after an overnight fast, in

32 randomized order. Subjects were cannulated and intubated with a gastric tube for both tests. For

33 test one, subjects ate $400 \mathrm{ml}$ soup with a spoon and for test two the soup was infused into the

34 stomach at an equivalent rate. Subsequently measurements of glycaemic (GR) and insulinaemic

35 responses (IR) from cannula samples, breath samples for measurement of gastric emptying using

36 the $\left[{ }^{13} \mathrm{C}\right]$ sodium acetate breath test and visual analogue scales (VAS) for satiety were taken over

37180 minutes. There were differences in IR over the first 15 minutes (Oral: 169.0 \pm 22.1 ; Gastric

$38124.1 \pm 18.8 ; \mathrm{t}(9)=2.67 ; \mathrm{p}=0.028)$ but no difference in GR. There were differences in gastric

39 emptying half time (Oral: $85.0 \pm 2.7$; Gastric $79.4 \pm 3.3 ; \mathrm{t}(9)=2.40 ; \mathrm{p}=0.04$ ) and ascension

40 time (Oral: $68.2 \pm 2.2 ;$ Gastric $64.0 \pm 2.2 ; \mathrm{t}(9)=2.57 ; \mathrm{p}=0.03$ ) with food taking longer to empty

41 from the stomach on the Oral test day than on the Gastric test day. There was no significant

42 difference in the satiety ratings. This study demonstrated that bypassing oro-pharyngeal and

43 oesophageal exposure decreases the normal physiological CPR with detriment to IR and gastric

44 emptying.

45

46 Keywords: glycaemic response, insulin, gastric emptying, cephalic, satiety 
49 Oral ingestion of food and beverages provides a sensory experience including anticipation, sight,

50 smell, chewing and taste. Bypassing this route removes much of this sensory stimulation and

51 therefore reduces the normal physiological cephalic phase responses (CPR). These responses

52 assist in early initiation of digestion and maximal absorption of nutrients prior to ingestion [1-3].

53 One response of particular interest is the CPR secretion of insulin (CPIR) and its role within

54 glycaemic control [4-8]. Oral stimulation by food initiates fast release of insulin; that peaks

55 between 1-4 minutes returning to baseline within ten minutes, the consequence of which avoids

56 both peak levels of glucose release and subsequent gluconeogenesis and lipolysis [1, 3, 9].

58 Work by Teff [10] identified that CPIR caused a 30\% reduction in plasma glucose post ingestion

59 of food and Ahren and Holst [6] found that blocking neural pathways for CPR caused a reduction

60 of $73 \%$ in CPIR, causing higher plasma glucose levels for longer following food ingestion.

61 Western diets currently contain more energy dense foods with less fibre [11]. These foods may

62 reduce oroosensory stimulation as they need less chewing and have a faster oral transit time [8].

63 This can result in a reduction in both CPRs and satiety [8, 12]. With the loss of oral sensory

64 stimulation and associated CPIR an individual's risk of hyperglycaemia and hyperinsulinaemia is

65 believed to be increased, and if this becomes a consistent metabolic profile there is a greater risk

66 of subsequent metabolic and cardio-vascular disease [12, 13]. To date, many of the studies have

67 focused on looking at the effect on glycaemic and insulinaemic responses directly following

68 sham feeding or oral stimulation feeding [14-16] for up $30 \mathrm{~min}$ but have not examined the 
69 impact of the CPIR on the entire postprandial period to demonstrate if they would present greater 70 metabolic risk.

72 Smeets et al. [8] believes that a loss of adequate oral sensory signalling from dietary choices that

73 are more readily available in western society today are responsible for a reduction in satiety.

74 Earlier work by Cecil et al. [14] identified that it is a combination of early oro-sensory

75 stimulation with gastro-intestinal influences such as motility and distension that provides the 76 greatest sensation of satiety. Furthermore utilising techniques that bypass oral CPR was found to

77 increase rates of gastric emptying and decrease satiety [14]. Increasing rates of gastric emptying

78 is likely to impact on satiety as food will remain in the stomach for a shorter time, decreasing the

79 time that the stomach remains distended which could result in increased food consumption [14, 80 17].

82 Although there is much evidence that the loss of CPIR impacts on the metabolic profile of an

83 individual there is some contradiction within the evidence [3-6, 8, 13]. Only recently have

84 studies combining sensory signals and the implications on food consumption been undertaken

85 [16]. Smeets et al. [8] identifies a need for further work to examine the impact of sensory signals 86 on both short term metabolic pathways and satiety. Previous work has primarily focused on the 87 effect of CPR on satiety and gastric emptying $[14,15]$ or on the role of CPR on immediate 88 insulinaemic responses (IR) and glycaemic responses (GR) [16] but has not looked at the 89 responses of both over the entire postprandial period where it is more likely to have a metabolic 90 implication. This study therefore aimed to examine two components of CPR in combination, 91 which is lacking from the extant literature. The first component that was measured was the effect 
92 of oral stimulation on IR and GR. The second component measured was changes in gastric

93 emptying and satiety.

94

95 Materials and Methods

97 Subjects

98 Twelve healthy males not suffering from diabetes or pre-diabetes were recruited for the study by

99 means of advertisements and personal communications. Volunteers were given full details of the

100 study protocol prior to giving their written informed consent. Ethical approval for this study was

101 provided by the Research Officer for Faculty of Health and Life Sciences, Oxford Brookes

102 University, UK, in accordance with the Declaration of Helsinki. All subject's fasting blood

103 glucose was $<6.1 \mathrm{mmol} / \mathrm{l}$ and their BMI was between $18.5-30 \mathrm{~kg} / \mathrm{m}^{2}$. None of the subjects were

104 taking any medication that would interfere with glucose metabolism or insulin signalling. None

105 of the participants were smokers. Eating behaviour was determined using the Three Factor

106 Eating Questionnaire [18]. Only those who did not consciously restrain their food intake due to

107 psychological reasons, weight concerns and external stimuli were included in the study. All

108 subjects were asked to rate their liking of the soup on a scale of 1-10 with 1 being strongly

109 disliking it. All volunteers reported liking the soup and rated it as 7 or greater and hence were

110 included in the study.

111

\section{Study Design}

113 The study required volunteers to attend the lab on three separate occasions. The first was for

114 initial preliminary assessment and if volunteers were suitable to proceed with the study they were 
115 required to attend for two subsequent test days; one consisting of oral ingestion of soup (Oral)

116 and one consisting of gastric infusion of the soup into the stomach via a feeding tube (Gastric).

117 The two tests days were carried out in random order, with a minimum of three, maximum seven

118 days between tests.

120 Subjects were requested to record their food intake in a weighed food diary the night before

121 attending for their first test and repeat this prior to their second test. They were requested to fast

122 from 22.00 the night before although were able to drink water. Subjects were also requested to

123 abstain from strenuous exercise and alcohol consumption the day before testing.

125 Preliminary assessment

126 Volunteers were requested to attend the lab after an overnight fast so a fasting blood glucose

127 measurement could be taken via a fingerprick blood sample (HemoCue 201+ glucose analyzer,

128 Angelholm, Sweden). A health questionnaire pertaining to food allergies and intolerances and

129 any known metabolic conditions or medication was requested. Baseline anthropometric

130 measurements of height, weight, body mass index (BMI) and blood pressure were undertaken

131 from each individual subject. These aimed to screen for any medical conditions or medication

132 that may interfere with glucose metabolism or insulin signalling; in which case the volunteer

133 would be declined from participating further within the study.

135 The preliminary test required volunteers to attempt insertion of an oral gastric feeding tube

136 (Vygon, 14 French Levine, length 125, Gastro-duodenal feeding tube, Ecouca, France). This

137 ensured volunteers could satisfactorily undertake the procedure and familiarise themselves with 
138 the technique. Self-insertion of the tube makes the process less stressful for the volunteer as it

139 gives them control over the rate of insertion [19]. Tube insertion was required to a length of 50-

$14055 \mathrm{~cm}$ and subjects needed to retain the tube placement comfortably. For subjects having

141 difficulty, repeated attempts were not encouraged as this can impact on the gastric response.

142 Accurate placement of the tube was confirmed by inserting $100 \mathrm{ml}$ of water and using a $100 \mathrm{ml}$

143 syringe to aspirate the gastric contents. Once $80 \%$ of this can be aspirated then the tube was

144 deemed to be in the stomach [20]. The gastric tube was then removed. Following preliminary

145 testing, two volunteers were unable to pass the oral gastric tube (gastric intubation) and

146 withdrew, leaving a subject group of 10 male participants $(37.8 \pm 3.4$ years; $1.77 \pm 0.03 \mathrm{~m}$;

$147 \quad 75.4 \pm 4.2 \mathrm{~kg})$.

149 Subjects who were able to proceed with the study were then timed whilst ingesting $400 \mathrm{ml}$ of the

150 test soup orally in order to measure the rate of normal feeding. This allowed determination of an

151 individual flow rate in $\mathrm{ml} / \mathrm{min}$ for gastric tube infusion of the soup using a syringe during the

152 Gastric test session.

154 Experimental Test Protocol.

155 Subjects attended the lab in the morning between 7-8am after an overnight fasting. On arrival, 156 cannulation of a superficial vein of the upper limb was undertaken to provide collection of blood

157 samples for glucose and insulin measurement throughout the test protocol. Samples were taken at 158 a baseline of -5 minutes, following the onset of soup ingestion / infusion at 3 minute intervals 159 for first 15 minutes and then subsequently at 15 minute intervals for a total of 180 minutes. 
160 Baseline assessment of satiety using 100mm visual analogue scale (VAS) and breath samples for 161 measurement of gastric emptying were also undertaken at -5 minutes.

163 Subjects inserted the oral gastric tube for both the Oral and the Gastric test in order to ensure 164 there was no physiological difference between circumstances of each test that may influence 165 findings. The oral gastric tube was removed by the subjects after completion of the first 15 166 minutes of the trial.

168 The $400 \mathrm{ml}$ of test soup was ingested orally by the subject or infused via the oral gastric tube at 169 the rate of normal eating as determined from the pre-test trial. The test meal and quantity were 170 based on previous similar studies $[14,15]$. Timing of each test trial began at the point of 171 initiating food eating / infusion. The test soup (Campbell's Cup Soup, Cream of tomato, Leeds, $172 \mathrm{UK}$ ) contained $25 \mathrm{~g}$ of available carbohydrate and had $100 \mathrm{mg}$ of $\left[{ }^{13} \mathrm{C}\right]$ labelled sodium acetate 173 added. This is a naturally stable carbon isotope which is rapidly absorbed and oxidised within the 174 liver to form labelled $\mathrm{CO}_{2}$ which subjects then exhale. The soup contained $170 \mathrm{kcal}, 2.5 \mathrm{~g}$ 175 protein, $28.3 \mathrm{~g}$ total carbohydrate, $4.9 \mathrm{~g}$ fat and $0.7 \mathrm{~g}$ sodium. The soup was prepared by adding 176 the soup powder to $300 \mathrm{ml}$ of boiling water, $100 \mathrm{ml}$ of water at room temperature and serving or 177 intubating immediately.

\section{Gastric emptying}

180 Collection of breath via a straw tube into a glass vial (10ml Exetainer, Labco, Bucks, UK) allows

181 analysis at a later stage as an indicator of the rate of gastric emptying. Work by Braden et al. [21] 182 identified that this non-invasive method is reliable, safe and cost-effective for measuring rates of 
183 gastric emptying. Subject's breath samples were taken at baseline of -5 minutes, postprandial at

$1843,6,9,12,15$ minutes and then every 15 minutes until 180 minutes. Samples were then analysed 185 using isotope ratio mass spectrometry (ABCA, SerCon Limited, Crewe, UK) and results were 186 expressed relative to V-PDB, an international standard for known ${ }^{13} \mathrm{C}$ composition. For breath

$187{ }^{13} \mathrm{CO}_{2}$ levels using 130 known standard samples, the coefficient of variation across these samples 188 has been shown to be $0.0044 \%[22] .{ }^{13} \mathrm{CO}_{2}$ values were expressed as the excess amount in the 189 breath above baseline and converted into moles. Data are then displayed as percentage of ${ }^{13} \mathrm{CO}_{2}$ 190 dose recovered per hour and cumulative percentage ${ }^{13} \mathrm{CO}_{2}$ recovered over time. $\mathrm{CO}_{2}$ production 191 was assumed to be $300 \mathrm{mmol} / \mathrm{m}^{2}$ body surface area per hour. Body surface area was calculated 192 using a validated weight-height formula [23]. This was then fitted to a gastric emptying model 193 developed by Ghoos et al., [24]. For all the data, $\mathrm{r}^{2}$ coefficient between the modelled and raw 194 data was calculated and $\mathrm{r}^{2}>0.95$. From this model several parameters were measured. Lag phase 195 and half time were calculated using the formulae derived by Ghoos et al., [24]. Lag phase is the 196 time taken to maximal rate of ${ }^{13} \mathrm{CO}_{2}$ excretion [25] and is equivalent to the time of the inflection 197 point [26]. Half time is the time it takes $50 \%$ of the ${ }^{13} \mathrm{C}$ dose to be excreted [25]. Latency phase 198 [26] is the point of intersection of the tangent at the inflection point of the ${ }^{13} \mathrm{CO}_{2}$ excretion curve 199 representing an initial delay in the excretion curve. Ascension time [26] is the time course 200 between the latency phase and the half time representing a period of high ${ }^{13} \mathrm{CO}_{2}$-excretion rates. 201

\section{Blood glucose}

203 Blood samples were used to test for blood glucose and insulin at each time point. Blood glucose 204 was measured using the HemoCue ${ }^{\circledR} 201+$ Glucose analyzer (HemoCue Ltd, Dronfield, UK). The $205 \mathrm{HemoCue}^{\circledR}$ is a reliable method of blood glucose analysis [27]. The laboratory's CV for 20 or 
206 more duplicate measurements of fasting glucose (i.e. minute-to-minute variation in human

207 subjects) was $<5 \%$. The inter-assay CV (i.e. analytical variation) on standard solutions was

$208<3.6 \%$.

$210 \quad$ Insulin

211 At each time point, $6 \mathrm{~mL}$ was collected into blood collection tubes treated with di Potassium

212 EDTA (BD vacutainer, Oxford, UK) and immediately stored in crushed ice. Samples were then

213 centrifuged $4000 \mathrm{rpm}, 4^{\circ} \mathrm{C}$, for 10 minutes. Plasma was removed and stored at $-40{ }^{\circ} \mathrm{C}$ until

214 analysis. Insulin concentrations in the plasma samples were determined by

215 electrochemiluminescence immunoassay using an automated analyzer (Cobas ${ }^{\circledR}$ E411; Roche

216 diagnostics, Burgess Hill, UK). The Cobas ${ }^{\circledR}$ system is a reliable method of blood insulin

217 determination [28]. The unit of measurement was $\mu \mathrm{U} / \mathrm{ml}$.

218

219 On completion of the 180 minutes testing, the subject's intra-venous cannula was removed.

221 Visual analogue scales

222 Throughout the test trials $100 \mathrm{~mm}$ visual analogue scale (VAS) were utilised by each subject; at

223 baseline, 6, and 15 minutes and then every subsequent 15 minutes for a total of 180 minutes in

224 order to gain some comparison between oral ingestion and gastric infusion on their desire to eat

225 and level of satiety. Each time point required subjects to make a vertical mark across the

226 horizontal VAS line with anchor points of 'not at all to 'extremely' for specific questions to rate

227 their level of hunger, fullness and desire to eat and anchor points of 'nothing at all' to 'large 
228 amount' for how much food they thought they could eat. Use of VAS as a reliable measure of

229 subjective appetite and predictability of feeding behaviour is validated by Sorensen et al. [29].

231 Statistical analysis

232 Results of blood glucose, insulin and VAS data were converted to reflect the change in GR, IR

233 and VAS respectively by subtracting the baseline value from those taken at set time points. It

234 was this change response value that was then used within all subsequent analysis. Incremental

235 area under the curve (IAUC) using the trapezoidal rule [30] was calculated for all GR, IR and

236 the four parameters of the VAS. GR and IR IAUC were calculated for the first $15 \mathrm{~min}$ of the test,

237 the first $60 \mathrm{~min}$ of the test and the entire 180 in of the test. Statistical analysis was undertaken

238 using Statistical Package for Social Sciences (SPSS, version 19.0, USA). Mean differences

239 between Oral and Gastric IAUC for total GR, IR and the four parameters of the VAS were

240 analyzed using paired sample t-test and the effect size as calculated using Cohen's $d$. Paired

241 sample t-test was also used for comparison of gastric emptying times. Results are expressed as

242 means \pm standard error (SE) unless otherwise stated and significance was defined as $\mathrm{p}<0.05$.

244 Results

246 Glycaemic Response

247 During the 180 minute test the GR peaked at 30 minutes in both Oral $(2.35 \pm 0.25 \mathrm{mmol} / \mathrm{L})$ and

248 Gastric $(2.76 \pm 0.35 \mathrm{mmol} / \mathrm{L})$ tests (Figure 1). In both tests, this peak was followed by a rapid

249 decline in glucose concentration with the nadir occurring at 75 minutes in the Oral test (- 
251 difference in the peak GR between the tests $(\mathrm{t}(9)=1.13 ; \mathrm{p}=0.29 ; d=0.50)$.

252

253 There were no significant differences in GR IAUC following either 180 min (Oral: 71.3 \pm 9.5

$254 \mathrm{mmol} / \mathrm{L} \cdot \mathrm{min} ;$ Gastric $79.7 \pm 12.7 \mathrm{mmol} / \mathrm{L} \cdot \mathrm{min} ; \mathrm{t}(9)=0.68 ; \mathrm{p}=0.51 ; d=0.24), 60 \mathrm{~min}($ Oral:

$25564.9 \pm 9.5 \mathrm{mmol} / \mathrm{L} \cdot \mathrm{min} ;$ Gastric $74.7 \pm 10.4 \mathrm{mmol} / \mathrm{L} \cdot \min ; \mathrm{t}(9)=0.78 ; \mathrm{p}=0.45 ; d=0.04)$ or $15 \mathrm{~min}$

256 (Oral: $6.0 \pm 1.2 \mathrm{mmol} / \mathrm{L} \cdot \mathrm{min} ;$ Gastric $6.1 \pm 1.7 \mathrm{mmol} / \mathrm{L} \cdot \min ; \mathrm{t}(9)=0.11 ; \mathrm{p}=0.91 ; d=0.31$ ).

258 Insulinaemic response

259 Over 180 minutes the IR (Figure 2) peaked at 30 minutes in both Oral $(51.8 \pm 8.1 \mathrm{uU} / \mathrm{ml}$ ) and

260 Gastric $(62.9 \pm 8.1 \mathrm{uU} / \mathrm{ml})$ tests with a nadir at 165 minutes for Oral $(-0.5 \pm 0.5 \mathrm{uU} / \mathrm{ml})$ and at 135

261 minutes for Gastric $(-1.2 \pm 0.3 \mathrm{uU} / \mathrm{ml})$. There was no significant difference in the peak IR

262 between the tests $(\mathrm{t}(9)=1.23 ; \mathrm{p}=0.25 ; d=0.38)$.

264 There were no significant differences in IR AUC following $180 \mathrm{~min}$ (Oral: 1897.7 \pm 309.2

$265 \mathrm{uU} / \mathrm{ml} \cdot \mathrm{min}$; Gastric $2037.5 \pm 205.9 \mathrm{uU} / \mathrm{ml} \cdot \mathrm{min}$; $(9)=0.61 ; \mathrm{p}=0.56 ; d=0.19)$ or $60 \mathrm{~min}($ Oral:

$2661638.7 \pm 262.0 \mathrm{uU} / \mathrm{ml} \cdot \mathrm{min}$; Gastric $1870.5 \pm 196.8 \mathrm{uU} / \mathrm{ml} \cdot \min ; \mathrm{t}(9)=1.10 ; \mathrm{p}=0.30 ; d=0.36)$ but

267 there was a difference after $15 \mathrm{~min}$, with the Oral test causing the greater IR (Oral: 169.0 \pm 22.1

$268 \mathrm{uU} / \mathrm{ml} \cdot \mathrm{min} ;$ Gastric $124.1 \pm 18.8 \mathrm{uU} / \mathrm{ml} \cdot \min ; \mathrm{t}(9)=2.67 ; \mathrm{p}=0.028 ; d=0.63)$.

\section{Gastric emptying}

271 There were significant differences in gastric emptying times between the meals for half time $272(\mathrm{t}(9)=2.40 ; \mathrm{p}=0.04)$ and ascension time $(\mathrm{t}(9)=2.57 ; \mathrm{p}=0.03)$ but not for latency or lag phase 
273 ( $p>0.05$ ). The food took $\sim 4-5$ min longer to empty from the stomach on the Oral test day than on

274 the Gastric test day (Table 1)

275

276 Satiety

277 There were no significant differences in satiety ratings following the two different feeding

278 methods for any of the parameters hunger $(\mathrm{t}(9)=0.38 ; \mathrm{p}=0.71)$, fullness $(\mathrm{t}(9)=0.96 ; \mathrm{p}=0.36)$,

279 desire to eat $(\mathrm{t}(9)=1.60 ; \mathrm{p}=0.15)$ and prospective consumption $(\mathrm{t}(9)=0.68 ; \mathrm{p}=0.52)$ (Table 2;

280 Figure 3).

281

282 Discussion

283

284 This study was the first to examine the entire IR and GR postprandial profile in combination with

285 GR and satiety following Oral and Gastric feeding. The study demonstrated no significant

286 differences between oral or intubated feeding on GR however the Oral method of feeding

287 resulted in a greater IR over the first 15 minutes. There were also no significant differences

288 found between Oral or Gastric feeding on satiety but gastric emptying was significantly

289 accelerated by $4-5 \mathrm{~min}$ on the Gastric test in comparison to the Oral test.

291 In comparing oral ingestion with intubated feeding it was hypothesised within the present study

292 that bypassing oro-pharyngeal and oesophageal exposure would decrease the CPIR [31].

293 Findings from this present study based on the first $15 \mathrm{~min}$ of IAUC insulin data appear to

294 replicate the characteristic early CPIR profile reported in previous work from oral ingestion of

295 food [10]. However there were no differences in GR between the oral ingestion compared with 
296 intubated feeding. Early CPIR occurs to a peak within the initial 1- 4 minutes after gustatory

297 stimulation returning to baseline within ten minutes [1-3, 9, 32]. Although overall it is a minimal

298 rise in insulin concentration levels compared to those secreted postprandially (5uU/ml [5] for

299 cephalic response compared to a postprandial response that could be $\sim 60 \mathrm{uU} / \mathrm{ml}$ (current study)),

300 it is believed to increase digestive secretions, decrease gut motility and decrease food intake [1-

$3013]$.

302

303 The effects of CPIR on blood glucose appear to differ. Findings in a study by Teff et al. [32]

304 identified that in normal weight healthy males, 4 minutes post ingestion of food there was a

305 significant increase in insulin and also a significant drop in plasma glucose as a result of early

306 CPIR. However as in the current study, this change in GR has not been replicated in all studies

307 perhaps due to difficulty in measuring such small variations in blood samples [5, 8]. Ranawana

308 et al. [33] also identified high variability in glucose absorption between individuals even after

309 eating the same food. This presents some evidence that within-individual variance is also a

310 possibility even in a controlled methodology which may account for the lack of differences seen

311 here. A further consideration is that simultaneous release of glucagon during CPR may prevent a

312 reduction in blood glucose levels caused by CPIR [5] but without measuring glucagon levels this

313 is an unknown factor in the present study.

315 Other reasons for disparity within the results of studies on CPR maybe palatability and the

316 duration of oral transit time [8]. To date there is mixed findings as to whether palatability

317 directly impacts vagally activated pathways to increase concentrations of CPIR; which may

318 influence study findings if there is inconsistency within the subject group [9, 29, 34]. However 
319 within the present study an initial questionnaire undertaken identified that none of the subjects

320 had a dislike for the tomato soup, utilised. Expert opinion is also divided in relation to the effect

321 the texture or form of food choice may have on oral exposure time. Teff [34] believes there is a

322 lack of CPIR to liquid stimuli; suggesting that chewing is required for adequate vagal stimulation

323 for insulin secretion. However, Cecil et al. [14, 15, 35] validate the use of liquid soup for

324 initiating CPR where they found the influence of sight, smell, and taste played an important part

325 in stimulating both pancreatic and gastric secretions as well as influencing appetite regulatory

326 centres. Cassady et al. [36] and deGraaf [12] also identify that liquid such as soup when eaten

327 with a spoon extends oro-sensory transit time to increase both CPR and satiety. A final

328 explanation for the differences in results seen between studies may come from work by Cecil et

329 al. [15], who found that the macronutrient content of the soup plays an important role as high fat

330 soup suppressed hunger, induced fullness, and slowed gastric emptying more than the high-

331 carbohydrate soup when ingested orally but there was no differences between the soups when

332 they were given intragastically.

334 One of the main aims of the current research was to combine satiety and metabolic responses

335 within the one study. Oro-sensory stimulation has been shown to increase the secretion of gastro-

336 intestinal peptides, these peptides slow gastric emptying [5,34]. The delay in gastric emptying

337 increases satiety by prolonging stomach distention $[8,14,31]$. The present study hypothesised

338 that bypassing oro-pharyngeal and oesophageal exposure would reduce levels of satiety by

339 reducing the insulinaemic response and accelerating gastric emptying. Bypassing oro-pharyngeal

340 and oesophageal exposure did result in a slight acceleration in gastric emptying and increased

341 insulinaemic response however it was not possible to detect any differences from this in satiety. 
342 A recent study [16] was able to confirm that gastric infusion of nutrients induced greater appetite

343 ratings than ingestion, alongside increases in satiety hormones however they were unable to

344 detect changes in food intake. Cecil et al. [14, 15, 35] also utilised gastric intubation in a series of

345 studies assessing the impact of bypassing oro-pharyngeal stimulation on satiety and gastric

346 emptying. They identified that loss of oro-sensory stimulation impacted negatively on both

347 satiety and gastric emptying, with subjects feeling fuller earlier with greater suppression of

348 hunger when food was eaten orally. The present study failed to find significant differences

349 between the tests for satiety; this may be due to the gastric emptying changes of only $4-5 \mathrm{~min}$,

350 which although significant may not be sufficient to decrease satiety. It may also be due to the

351 large variability on the VAS data. However it should be noted that VAS assessments are only a

352 measure of perceived hunger not actual food intake and an ad libitum test meal would have

353 provided objective assessment of subsequent food intake [36, 37]. Although other studies have

354 been able to identify changes in VAS with oral stimulation using a similar sample size they have

355 not been able to detect changes in actual food intake [16]. An increased sample size however is

356 hindered by the invasive nature of the intubation test procedure.

\section{Conclusion}

360 In conclusion this study was able to demonstrate that utilising an oral gastric tube for infusion of

361 food to bypass oro-pharyngeal and oesophageal exposure decreases the normal physiological

362 CPR with detriment to IR and marginal accelerations in gastric emptying but was unable to

363 demonstrate any impact on satiety and GR. Potential future research could use a solid test meal

364 and include an ad libitum test meal. 
366 Conflict of Interest Statement: There are no conflicts of interest associated with this

367 manuscript

368

369 Acknowledgements: We are gratefully acknowledge Liz Kerrigan for her dedication, effort and

370 expertise in Isotope Ratio Mass Spectrometry analysis

371

372

373

374

375

376

377

378

379

380

381

382

383

384

385

386

387 
391 1. Zafra, M.A., F. Molina, and A. Puerto, The neural/cephalic phase reflexes in the

2. Power, M.L. and J. Schulkin, Anticipatory physiological regulation in feeding biology: physiology of nutrition. Neurosci Biobehav Rev, 2006. 30(7): p. 1032-44.

cephalic phase responses. Appetite, 2008. 50(2-3): p. 194-206.

3. Teff, K.L., How neural mediation of anticipatory and compensatory insulin release helps us tolerate food. Physiol Behav, 2011. 103(1): p. 44-50.

4. Teff, K.L. and K. Engelman, Palatability and dietary restraint: effect on cephalic phase insulin release in women. Physiol Behav, 1996. 60(2): p. 567-73.

5. Teff, K., Nutritional implications of the cephalic-phase reflexes: endocrine responses. Appetite, 2000. 34(2): p. 206-13.

6. Ahren, B. and J.J. Holst, The cephalic insulin response to meal ingestion in humans is dependent on both cholinergic and noncholinergic mechanisms and is important for postprandial glycemia. Diabetes, 2001. 50(5): p. 1030-8.

7. $\quad$ Fukaya, M., et al., Mechanism of rapid-phase insulin response to elevation of portal glucose concentration. Am J Physiol Endocrinol Metab, 2007. 293(2): p. E515-22.

8. Smeets, P.A., A. Erkner, and C. de Graaf, Cephalic phase responses and appetite. Nutr Rev, 2010. 68(11): p. 643-55.

9. Just, T., et al., Cephalic phase insulin release in healthy humans after taste stimulation? Appetite, 2008. 51(3): p. 622-7.

10. Teff, K.L. and K. Engelman, Oral sensory stimulation improves glucose tolerance in humans: effects on insulin, C-peptide, and glucagon. Am J Physiol, 1996. 270(6 Pt 2): p. R1371-9.

11. Stubbs, R.J., N. Mazlan, and S. Whybrow, Carbohydrates, appetite and feeding behaviour in humans. J Nutr, 2001. 131: p. 2775s-2781s.

12. de Graaf, C., Texture and satiation: the role of oro-sensory exposure time. Physiol Behav, 2012. 107(4): p. 496-501.

13. Malik, V.S., et al., Sugar-sweetened beverages and risk of metabolic syndrome and type 2 diabetes: a meta-analysis. Diabetes Care, 2010. 33(11): p. 2477-83.

14. Cecil, J.E., J. Francis, and N.W. Read, Relative contributions of intestinal, gastric, orosensory influences and information to changes in appetite induced by the same liquid meal. Appetite, 1998. 31(3): p. 377-90.

15. Cecil, J.E., J. Francis, and N.W. Read, Comparison of the effects of a high-fat and highcarbohydrate soup delivered orally and intragastrically on gastric emptying, appetite, and eating behaviour. Physiol Behav, 1999. 67(2): p. 299-306.

16. Spetter, M.S., et al., Taste matters - effects of bypassing oral stimulation on hormone and appetite responses. Physiol Behav, 2014. 137: p. 9-17.

17. Geliebter, A., Gastric distension and gastric capacity in relation to food intake in humans. Physiol Behav, 1988. 44(4-5): p. 665-8.

18. Stunkard, A.J. and S. Messick, The three-factor eating questionnaire to measure dietary restraint, disinhibition and hunger. J Psychosom Res, 1985. 29(1): p. 71-83.

19. Leiper, J.B., et al., The effect of intermittent high-intensity running on gastric emptying of fluids in man. Med Sci Sports Exerc, 2005. 37(2): p. 240-7. 
20. Beckers, E.J., et al., Determination of total gastric volume, gastric secretion and residual meal using the double sampling technique of George. Gut, 1988. 29(12): p. 1725-9.

21. Braden, B., et al., The [13C]acetate breath test accurately reflects gastric emptying of liquids in both liquid and semisolid test meals. Gastroenterology, 1995. 108(4): p. 104855 .

22. Clegg, M.E. and A. Shafat, Procedures in the 13C octanoic acid breath test for measurement of gastric emptying: analysis using Bland-Altman methods. Scand $\mathbf{J}$ Gastroenterol, 2010. 45(7-8): p. 852-61.

23. Haycock, G.B., G.J. Schwartz, and D.H. Wisotsky, Geometric method for measuring body surface area: a height-weight formula validated in infants, children, and adults. J Pediatr, 1978. 93(1): p. 62-6.

24. Ghoos, Y.F., et al., Measurement of gastric emptying rate of solids by means of a carbonlabeled octanoic acid breath test. Gastroenterology, 1993. 104(6): p. 1640-7.

25. Jackson, S.J., L.J. Bluck, and W.A. Coward, Use of isotopically labelled octanoic acid to assess the effect of meal size on gastric emptying. Rapid Commun Mass Spectrom, 2004. 18(10): p. 1003-7.

26. Schommartz, B., D. Ziegler, and P. Schadewaldt, Significance of diagnostic parameters in [13C]octanoic acid gastric emptying breath tests. Isotopes Environ Health Stud, 1998. 34(1-2): p. 135-43.

27. Stork, A.D., et al., Comparison of the accuracy of the HemoCue glucose analyzer with the Yellow Springs Instrument glucose oxidase analyzer, particularly in hypoglycemia. Eur J Endocrinol, 2005. 153(2): p. 275-81.

28. Siahanidou, T., et al., Circulating visfatin levels in healthy preterm infants are independently associated with high-density lipoprotein cholesterol levels and dietary long-chain polyunsaturated fatty acids. Metabolism, 2010. 60(3): p. 389-93.

29. Sorensen, L.B., et al., Effect of sensory perception of foods on appetite and food intake: a review of studies on humans. Int J Obes Relat Metab Disord, 2003. 27(10): p. 1152-66.

30. Brouns, F., et al., Glycaemic index methodology. Nutr Res Rev, 2005. 18(1): p. 145-71.

31. Stratton, R.J., R.J. Stubbs, and M. Elia, Short-term continuous enteral tube feeding schedules did not suppress appetite and food intake in healthy men in a placebocontrolled trial. J Nutr, 2003. 133(8): p. 2570-6.

32. Teff, K.L., R.D. Mattes, and K. Engelman, Cephalic phase insulin release in normal weight males: verification and reliability. Am J Physiol, 1991. 261(4 Pt 1): p. E430-6.

33. Ranawana, V., et al., Postmastication digestion factors influence glycemic variability in humans. Nutr Res, 2011. 31(6): p. 452-9.

34. Teff, K.L., Cephalic phase pancreatic polypeptide responses to liquid and solid stimuli in humans. Physiol Behav, 2010. 99(3): p. 317-23.

35. Cecil, J.E., J. Francis, and N.W. Read, Investigation into the role of cephalic stimulation of acid secretion on gastric emptying and appetite following a soup meal using the gastric acid inhibitor omeprazole. Appetite, 2004. 42(1): p. 99-105.

36. Cassady, B.A., R.V. Considine, and R.D. Mattes, Beverage consumption, appetite, and energy intake: what did you expect? Am J Clin Nutr, 2012. 95(3): p. 587-93.

37. Hlebowicz, J., Postprandial blood glucose response in relation to gastric emptying and satiety in healthy subjects. Appetite, 2009. 53(2): p. 249-52. 


\section{$481 \quad$ List of figures:}

482

483 Figure 1. Change in blood glucose response (mean \pm standard error) following Oral and Gastric 484 tests over 180 minutes (a) and over 15 minutes (b).

485 a)

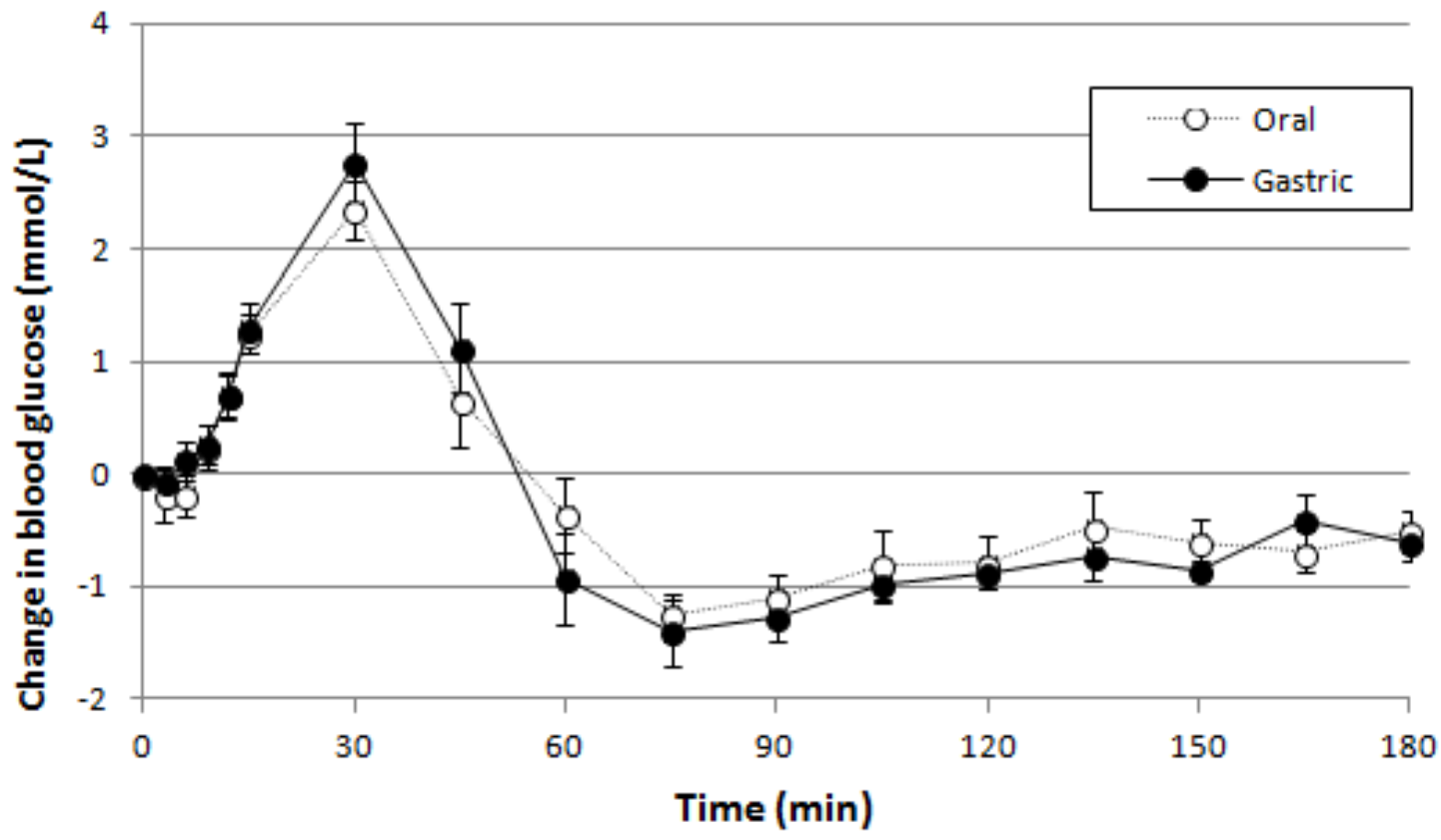




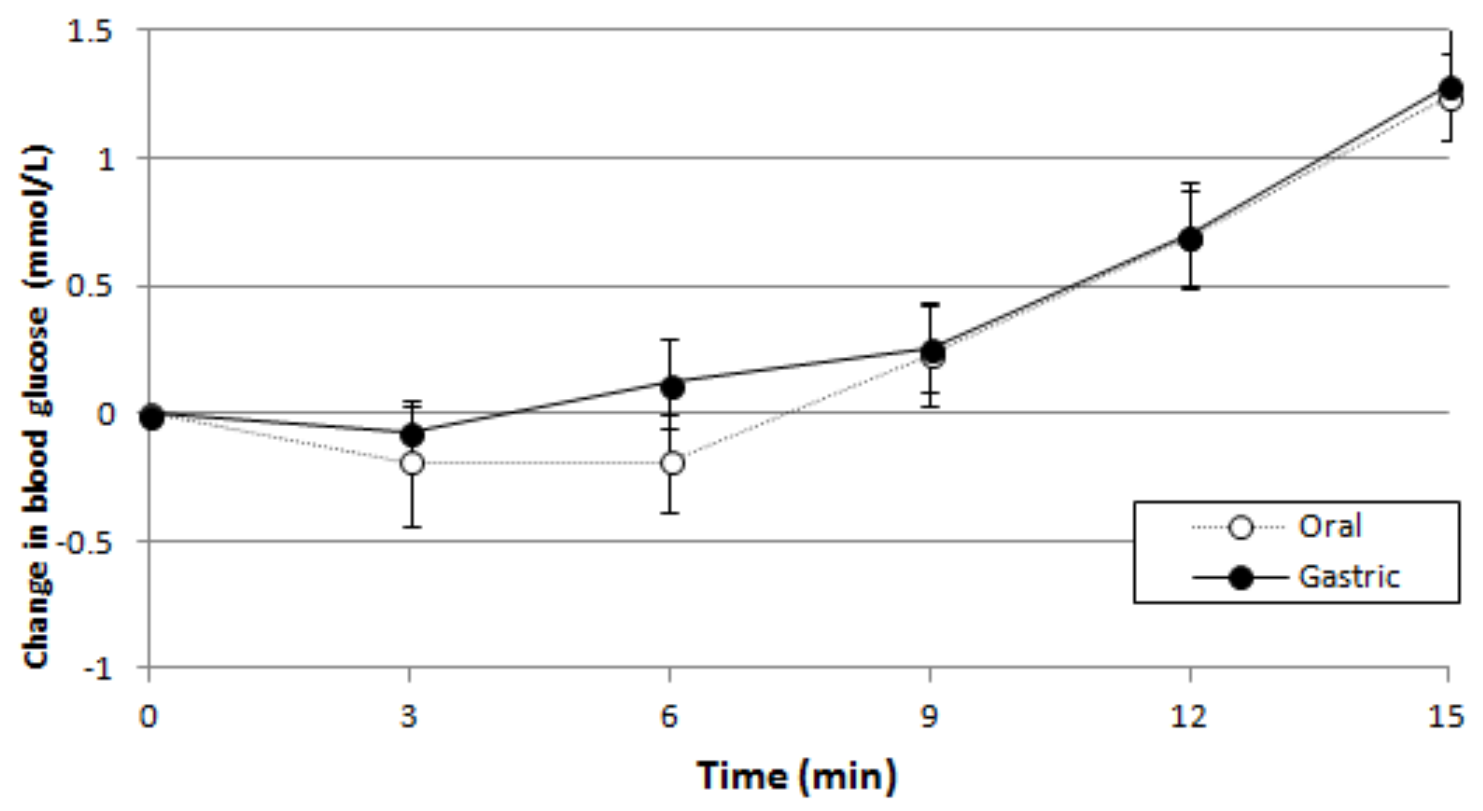

494

495

496

497

498

499

500

501

502

503

504

505

506

507

508 
509 Figure 2. Change in insulinaemic response (mean \pm standard error) following Oral and Gastric

510 tests over 180 minutes (a) and over 15 minutes (b).

511 a)

512

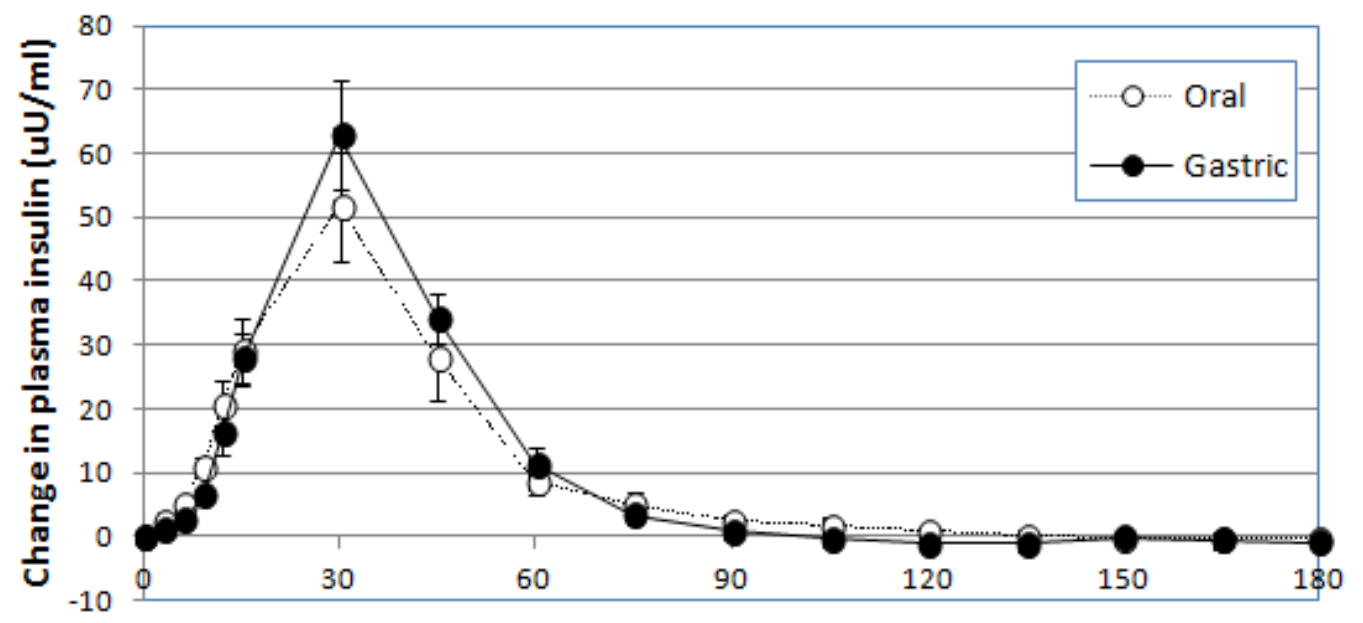

513

Time (min)

514 b)

515

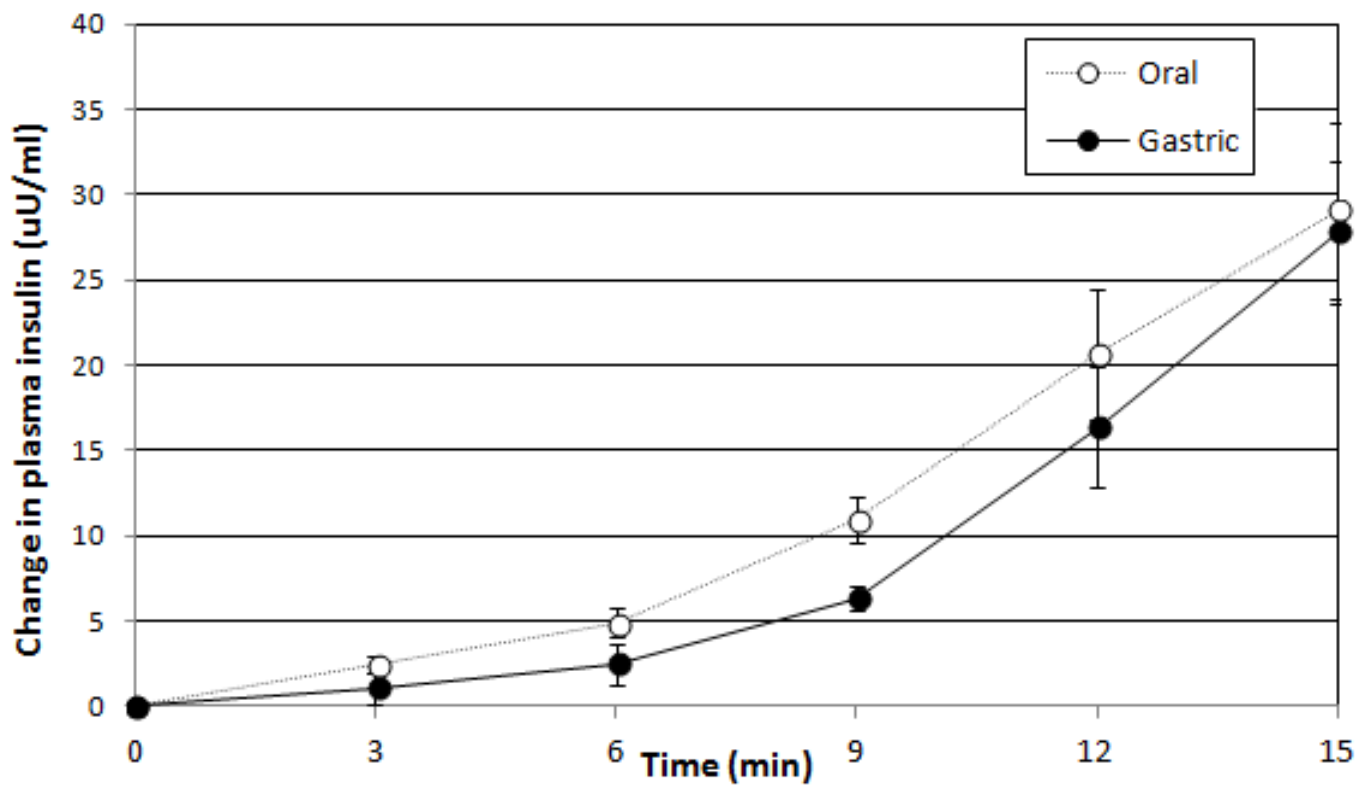

516 
518 Figure 3. Change in satiety ratings from visual analogue scales (mean \pm standard error) following

519 Oral and Gastric tests for hunger (a), fullness (b), desire to eat (c) and prospective consumption 520 (d).

521 a)

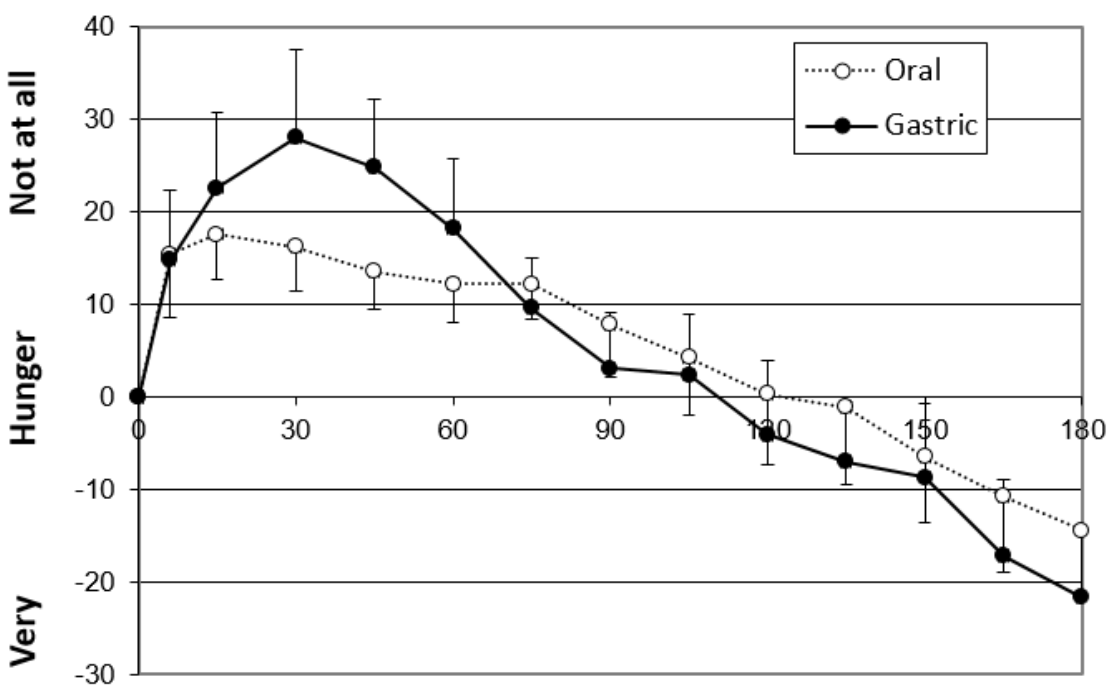

Time (min)

522

b)

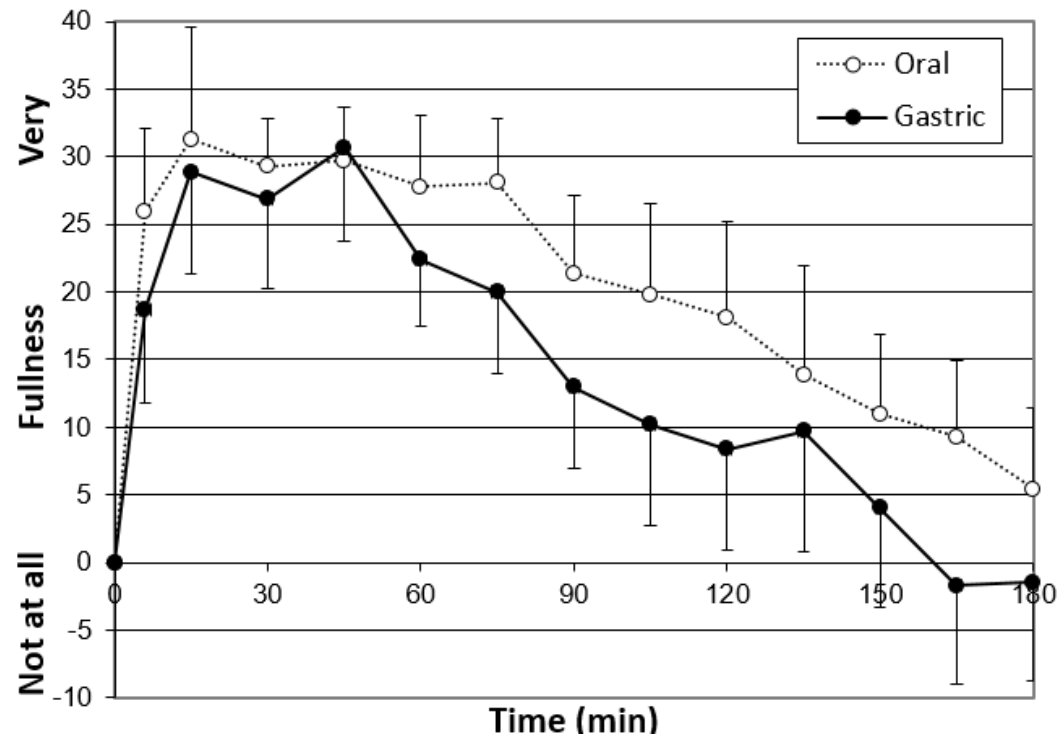


c)

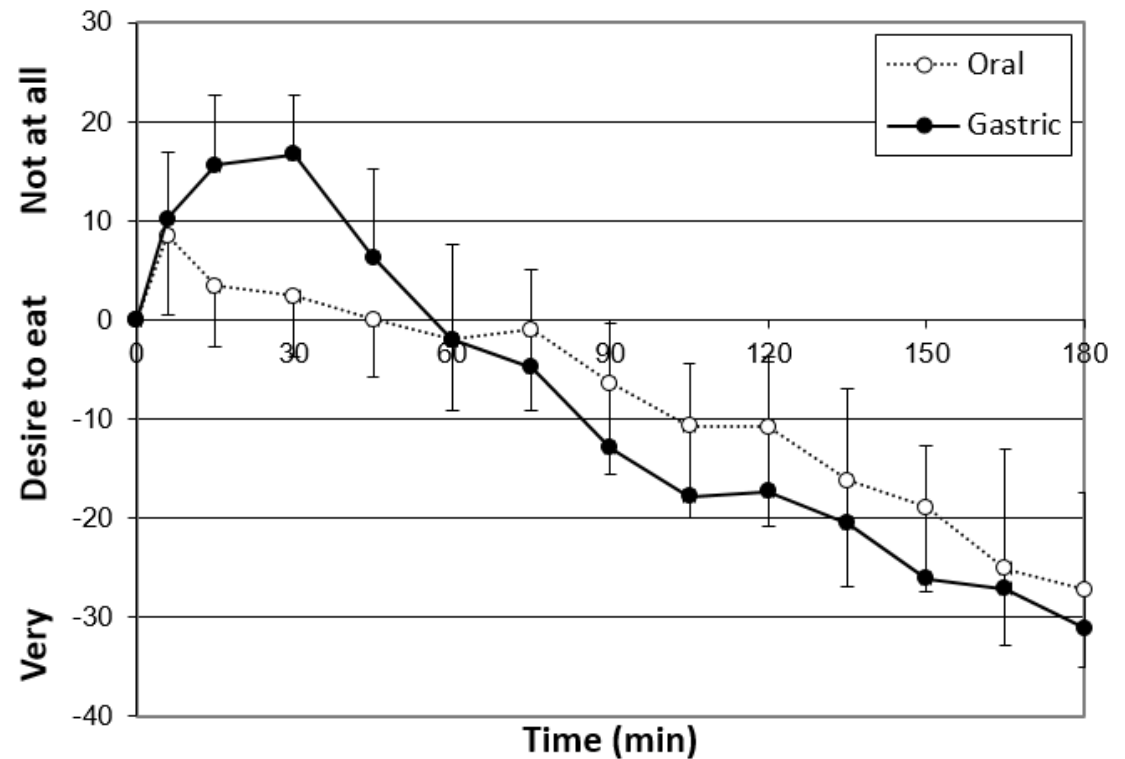

528

d)

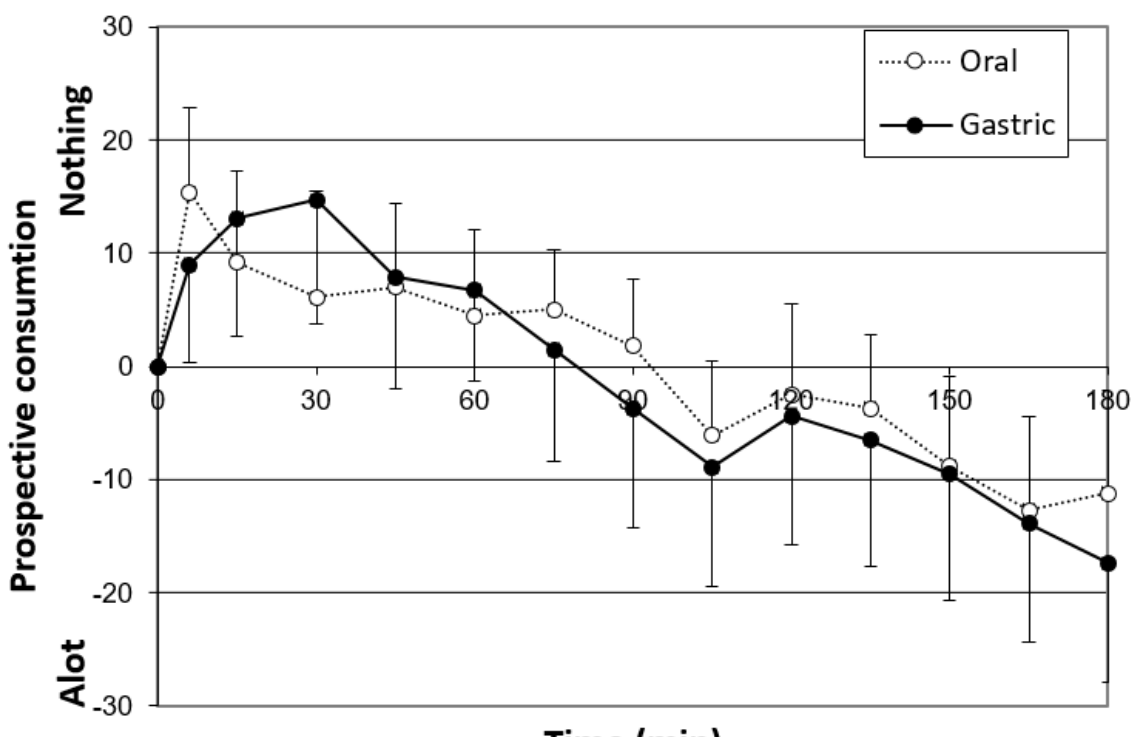




\section{$534 \quad$ List of Tables}

535

536 Table 1: Gastric emptying times (mean \pm standard error) following Oral and Gastric infusion of

537 soups. ${ }^{*}=\mathrm{p}<0.05$

\begin{tabular}{llll}
\hline Time & Oral & Gastric & $\boldsymbol{d}$ \\
\hline $\begin{array}{l}\text { Latency Phase } \\
\text { (min) }\end{array}$ & $16.8 \pm 0.7$ & $15.4 \pm 1.3$ & 0.44 \\
Lag phase (min) & $52.4 \pm 1.8$ & $48.4 \pm 2.8$ & 0.55 \\
Half time (min) & $85.0 \pm 2.7$ & $79.4 \pm 3.3^{*}$ & 0.59 \\
$\begin{array}{l}\text { Ascension time } \\
\text { (min) }\end{array}$ & $68.2 \pm 2.2$ & $64.0 \pm 2.2^{*}$ & 0.60 \\
\hline
\end{tabular}

538 
554 Table 2: Satiety rating, hunger, fullness, desire to eat and prospective consumption (mean \pm 555 standard error) following Oral and Gastric infusion test.

\begin{tabular}{llll}
\hline & Oral & Gastric & $\boldsymbol{d}$ \\
\hline Hunger (mm.min) & $1914 \pm 489$ & $2298 \pm 731$ & 0.20 \\
Fullness (mm.min) & $3964 \pm 711$ & $3330 \pm 808$ & 0.26 \\
Desire to eat (mm.min) & $1149 \pm 712$ & $2050 \pm 802$ & 0.38 \\
Prospective consumption & $1680 \pm 621$ & $2233 \pm 848$ & 0.24 \\
(mm.min) & & & \\
\hline
\end{tabular}

